\title{
Enterobius vermicularis in anterior chamber of eye
}

\author{
Kabindra Bajracharya ${ }^{1}$, Arjun Malla Bhari', Salma KC Rai ${ }^{2}$, Saraswati Pandey ${ }^{3}$ \\ ${ }^{1}$ Associate Professor and Consultant Pediatric Ophthalmologist, ${ }^{2}$ Professor and Consultant Pediatric Ophthalmologist, \\ ${ }^{3}$ Assistant Professor and Pediatric Ophthalmologist, Lumbini Eye Institute, Bhairahawa, Nepal
}

Enterobius vermiculari which is often referred as pinworm, is an intestinal nematode which is transmitted through fecal-oral route. The extraintestinal presentation of the worm is rare. A live worm, Enterobius vermicularis in anterior chamber is a very rare case. To present an unusual case of Enterobius vermicularis in anterior chamber of right eye in a 3 years old girl. Visual acuity assessment, slit lamp examination and ultrasonography of the eye were performed. The worm was found in anterior chamber of right eye at 8-9 o' clock hour position, coiling at the presentation. The living, white worm was freely moving and changing position frequently. There was hypopyon with exudates inferiorly. The pupil was irregular, posterior synechiae was present with cataractous lens. The living worm was removed surgically under general anesthesia and sent for microbiological examination. Synecholysis with lens aspiration and Posterior chamber intraocular lens implantation was done in second surgery. An adult worm in anterior chamber is rare. Treatment is surgical removal. The visual prognosis is not good when the case present with severe form of anterior uveitis and complicated cataract.

Access this article online

Website:

http://nepjol.info/index.php/AJMS DOI: 10.3126/ajms.v10i6.26000 E-ISSN: 2091-0576 P-ISSN: 2467-9100

Key words: Anterior chamber; Enterobius vermicualris; Complicated cataract

\section{INTRODUCTION}

Enterobius vermicularis, also known as pinworm, is an intestinal nematode which is transmitted through fecal-oral route. ${ }^{1}$ Adult worms live in the caecum and vermiform appendix of human. ${ }^{2}$ The extra intestinal presentation of the worm is rare. This case reports the presence of an adult live worm, E. vermicularis in anterior chamber of the eye in a 3 year-old girl. There are few published case reports with different types of worms found in anterior chamber of eye, Loa loa. ${ }^{3-5}$ Brugia malayi, ${ }^{6}$ Wuchereria Bancrofti, ${ }^{7}$ Angiostrongylus, ${ }^{8}$ Linguatula serrata. ${ }^{9-11}$ There are two published case report with E. vermicularis in ocular orbit of 14 year-old girl ${ }^{1}$ and a case with the worm found in conjunctival sac in 15 year-old girl $^{12}$ but reported cases of E. vermicularis in anterior chamber of eye in english literature is, probably the first case.

\section{CASE REPORT}

A 3 year-old girl from Balarampur, India was brought to our pediatric ophthalmology and strabismus department on $5^{\text {th }}$ February 2014 with complain of redness and watering of right eye since one and a half months. Her visual acuity was perception of light on right eye and CSM on left eye. She was photophobic on right eye. On slight lamp examination, live, white moving worm was found in anterior chamber of right eye at 8-9 o' clock position (Figures 1 and 2). There were membranous exudate inferiorly from 4-8 o' clock. The pupil was irregular and fixed, posterior synechiae was present with cataractous lens. The left eye was within normal limits.

B-scan of right eye was normal. Stool examination was negative for worms. Blood investigations $\mathrm{Hb}$ was $12.1 \mathrm{gm} \%$, $\mathrm{TC}=8900 \mathrm{cells} / \mathrm{cmm}, \mathrm{DC}-\mathrm{N}=50 \%, \mathrm{~L}=48 \%, \mathrm{M}=1 \%$, $\mathrm{E}=1 \%$. The emergency surgery was scheduled under general anesthesia.

\section{SURGICAL PROCEDURE}

Two clear corneal tunnel was made $2.8 \mathrm{~mm}$ at 9 o' clock with keratome and $1 \mathrm{~mm}$ with sideport knife at

\footnotetext{
Address for Correspondence:

Dr. Kabindra Bajracharya, Associate Professor and Consultant Pediatric Ophthalmologist, Lumbini Eye Institute, Siddharthanagar-3,

Bhairahawa, Nepal. Tel: +977-9841302529. E-mail: kabindra_baj@yahoo.com (c) Copyright AJMS
} 
3 o'clock. Ringer/lactate solution was used to irrigate and bring worm to side port incision (Figure 3). The worm was removed with the help of Mc Pherson forcep.

The table hyphema was noted. The anterior chamber was washed and formed, two nylon 10 ' 0 suture was applied and subconjunctival injection of dexamethasone and gentamycin given. The worm was kept in a bottle containing

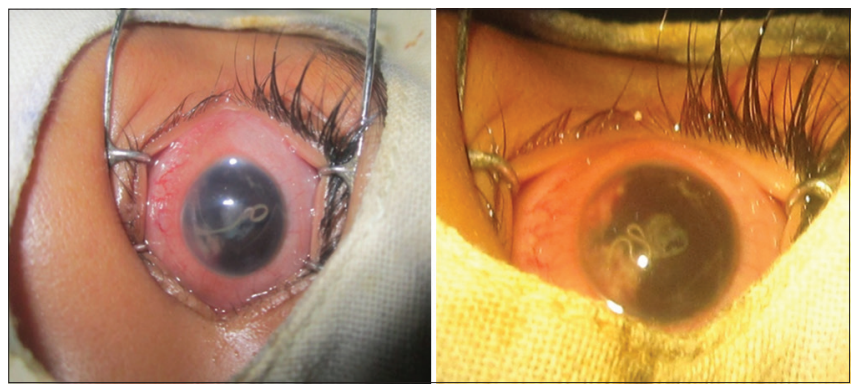

Figure 1 and 2: Depicting worm in the anterior chamber of right eye changing position

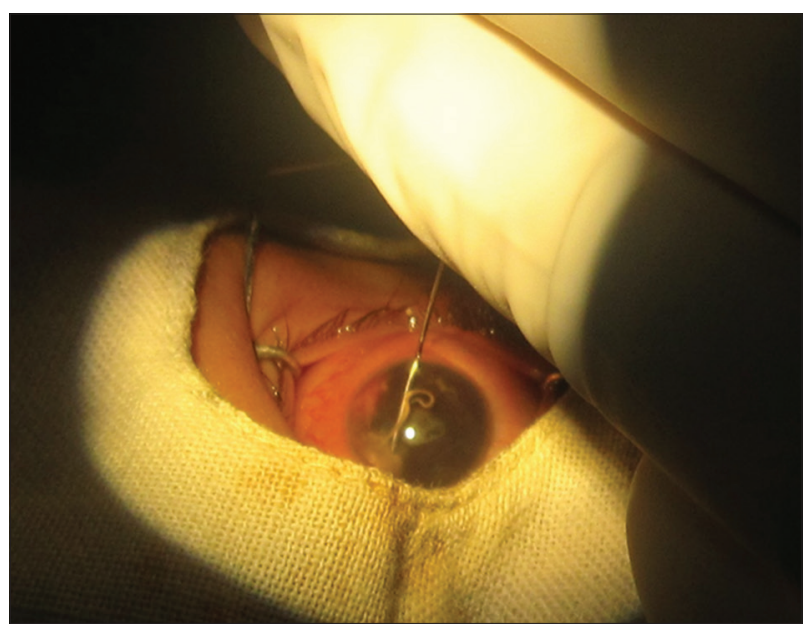

Figure 3: Irrigating worm with Ringer/Lactate solution

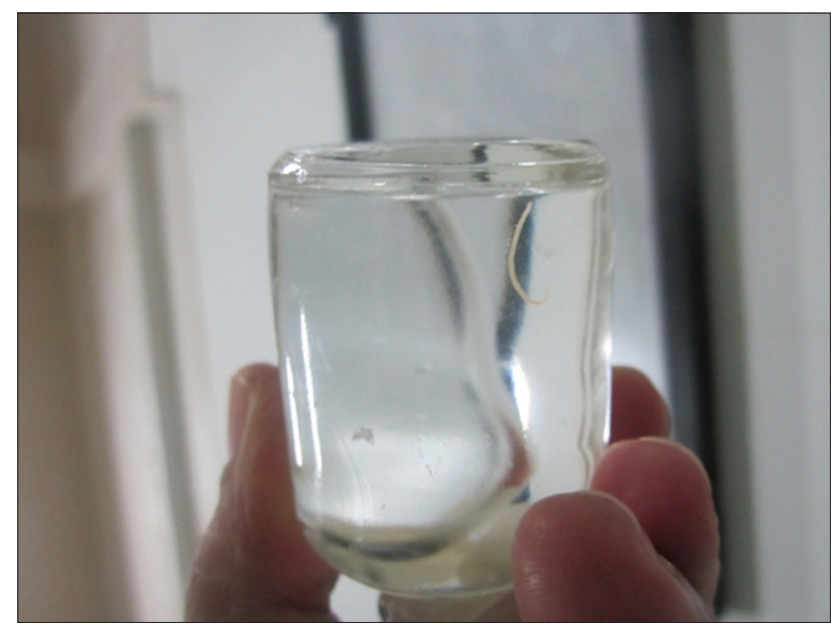

Figure 4: Retrieved worm in bottle containing normal saline normal saline and sent for microbiological examination at Microbiology department of Universal College of Medical Sciences, Bhairahawa (Figure 4). The worm was confirmed to be Enterobius vermicularis.

Postoperative treatment included prednisolone acetate eye drops six times per day, moxifloxacin eight times per day, homatropine twice a day and choramphenicol and dexamethasone ointment for night. The medication was tapered down and at two weeks postoperative day follow-up visit. Visual acuity was perception of light. Slit-lamp examination revealed 360 degree posterior synechiae, anterior membrane in front of lens with cataract.

Synecholysis with lens aspiration and posterior chamber intraocular lens implantation was done in second surgery under general anesthesia after six weeks of first surgery.

Again in subsequent follow up slit-lamp examination revealed thick posterior chamber opacification and membranectomy was planned but patient didn't come for surgery.

\section{DISCUSSION}

Enterobius vermicularis is cosmopolitian in distribution, being found all over the world.

\section{Life cycle}

The extraintestinal presentation of E. vermicularis is rare. Ocular presentation of the worm is even rare. In one reported case, in total six worms removed from right lower lid in the inferior conjunctival sac. There was also one worm crawling out of the nose. ${ }^{1}$ In next reported case 42 worms were expelled from eye, conjuctival sac in 3 weeks period. ${ }^{12}$ The mechanism by which eggs or worms reached the conjunctival sac was not clear but they suspected it was most likely the result of direct inoculation of adult female worms from perianal skin to the eyes by the child's fingers. Alternatively, eggs could have been inoculated, followed by hatching of both male and female worms. But our case was more complicated. The first likely mechanism might be direct inoculation of eggs which enter to the anterior chamber through conjunctival vessels and grown into adult form. Next possible mechanism might be hematogenous spread through long posterior ciliary artery or choroidal circulation carrying egg entered and lodged in anterior chamber. But the exact mechanism is not clear.

In two of the reported cases of E. vermicularis, anterior chamber and lens were unaffected, so they had better visual acuity. In our case, the patient presented with membraneous 
exudates, posterior synechiae with severe anterior uveitis and complicated cataract. The visual outcome was poor in the patient. In case of Babady N. Esther et al, ${ }^{1}$ stool examination was positive for E. vermicularis eggs but in case of Dutta LP it was negative. In our case no eggs were found in stool examination.

\section{CONCLUSION}

An adult worm in anterior chamber is rare. Treatment is surgical removal. The visual prognosis is bad when the case present late with severe anterior uveitis and complicated cataract.

\section{REFERENCES}

1. Babady N. Esther, Awender E, Geller R, Miller T, Scheetz G, Arguello $\mathrm{H}$, et al. Enterobius vermicularis in a 14- year-old Girl's Eye. J Clin Microbiol 2011; 49(12): 4369-4370.

2. Chatterjee KD. Enterobius vermicularis, p. Parasitology (Protozoology and Helminthology), $12^{\text {th }}$ ed. Chattarjee medical publishers, Calcutta 1980; p179-182.

3. Hassan S, Isyaku M, Yayo A, Fada FS, Lhesiulor GU and lliyasu G. Adult Loa Loa Filarial worm in the anterior chamber of the eye: a first report from Savanna Belt of Northern Nigeria. PLOS Neglected Tropical Diseases: 2016; 1-4.

4. Qazi MS, Khetan SP and Tankhiwale SS. An adult worm in the eye. IJMDS 2016; 5(2): 1269-1271.

5. Kagmeni G, Cheuteu R, Bilong $Y$ and Wiedemann P. Anterior chamber live Loa Loa: case report. Clinical Medical Insights 2016; 9: 55-56.

6. Mohan A, Verghese A, Raman M and Biswas J. Live Brugia malayi in the anterior chamber: a case report from India. Eye 2014; 28: 1038.

7. Khokhar S, Patil B, Sharma R, Sinha G, Gupta S, Kakkar P, et al. Live Wucherreria bancrofti in anterior chamber of the eye: a case report. Trop J Med Res 2015: 18: 45-47.

8. Neekhra A, Agashe AP, Todkar H, Trivedi HL, Jain R and Fedge S. A live worm in anterior chamber. 2001; 132-133.

9. Pal SS, Bhargava M, Kumar A, Mahajan N, Das S, Nandi K, et al. An unusual intraocular tongue worm in anterior chamber: a case report. Ocular Immunology and Inflammation 2011; 19(6): 442-443.

10. Deweese MW, Murrah WF and Caruthers SB. Case report of a tongue worm (Linguatula serrata) in the anterior chamber. Arch Ophthalmol 1962; 68(5): 587-589.

11. Bhende M, Abhisek, Biswas J, Raman M and Bhende PS. Linguatula serrata in the anterior chamber of the eye. Indian J Ophthalmol 2014; 62(12): 1159-1162.

12. Dutta LP and Kalita SN. Enterobius vermicularis in the human conjunctival sac. Indian J Opthalmol 1976: 24: 34-35.

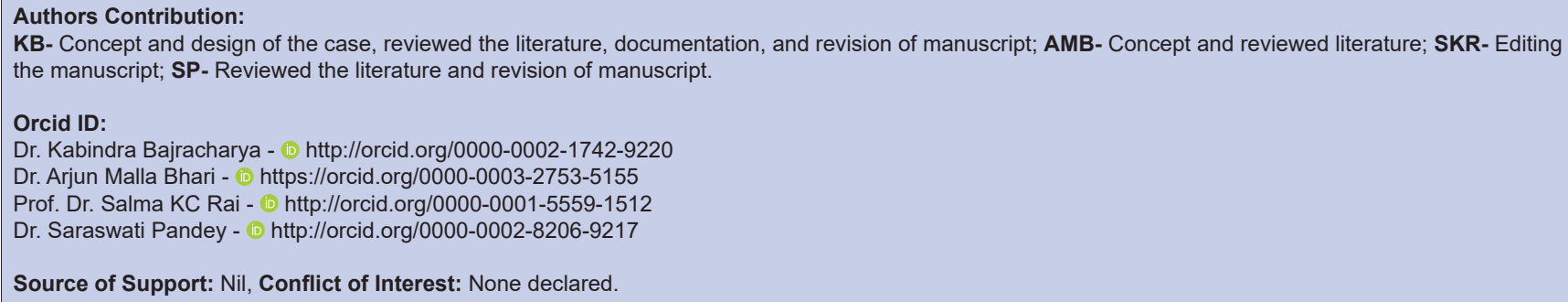

